\title{
Pentraxin 3 Released from Neutrophils Increases Plasma Levels in Patients with Acute Coronary Syndrome
}

\author{
Kenji Inoue, ${ }^{1}$ Satoru Suwa, ${ }^{2}$ Shinya Okazaki, ${ }^{1}$ Seigo Itoh, ${ }^{3}$ Alexander S. Savchenko, ${ }^{4}$ \\ Makoto Naito, ${ }^{4}$ Tatsuhiko Kodama, ${ }^{5}$ and Hiroyuki Daida ${ }^{3}$ \\ ${ }^{1}$ Department of Cardiology, Juntendo University Nerima Hospital, 3-1-10 Takanodai, Nerima-ku, Tokyo 177-8521, Japan \\ ${ }^{2}$ Department of Cardiology, Juntendo University Shizuoka Hospital, Izunokuni, Shizuoka 410-2295, Japan \\ ${ }^{3}$ Department of Cardiology, Juntendo University School of Medicine, Tokyo 113-8431, Japan \\ ${ }^{4}$ Division of Cellular and Molecular Pathology, Department of Cellular Function, Graduate School of Medical and Dental Sciences, \\ Niigata University, Niigata 951-8510, Japan \\ ${ }^{5}$ Laboratory for Systems Biology and Medicine, Research Center for Advanced Science and Technology, The University of Tokyo, \\ Tokyo 153-0041, Japan
}

Correspondence should be addressed to Kenji Inoue, inouelsbm@gmail.com

Received 10 August 2011; Accepted 26 September 2011

Academic Editor: A. Habib

Copyright (C) 2011 Kenji Inoue et al. This is an open access article distributed under the Creative Commons Attribution License, which permits unrestricted use, distribution, and reproduction in any medium, provided the original work is properly cited.

\begin{abstract}
Background. Our recently developed ELISA system for the detection of human pentraxin 3 (PTX3) in plasma has demonstrated that plasma PTX3 levels are increased in patients with unstable angina pectoris. However, the origin of the PTX3 from the ruptured plaque or a systemic process and which cells release PTX3 remain unclear. Methods. Blood samples were taken using an aspiration catheter from the site of the ruptured plaque and from the aorta during acute coronary interventions in 118 patients with acute coronary syndrome. These samples were analyzed for PTX3, and brain natriuretic peptide (BNP) was used as a control. Aspirated thrombi from patients with acute myocardial infarction (AMI) $(n=32)$ were examined by histological staining. Results. Plasma PTX3 levels were higher in blood samples taken from the site of plaque rupture compared to samples taken from the aorta $(5.61 \pm 1.91 \mathrm{ng} / \mathrm{mL}$ versus $4.72 \pm 5.61 \mathrm{ng} / \mathrm{mL}, P<0.05)$. On the other hand, BNP levels, as reference, were not different between the samples $(P=0.45)$. PTX3-positive neutrophils accounted for $70.4 \%$ of cells in harvested thrombi, with the remaining cells consisting of mononuclear cells. Conclusions. Infiltrating neutrophils in thrombi at the plaque rupture site are a diagnostically important source of PTX3 in patients with acute coronary syndrome.
\end{abstract}

\section{Introduction}

Pentraxin 3 (PTX3) is a long pentraxin, consisting of a Cterminal pentraxin module coupled with an unrelated $\mathrm{N}$ terminal domain [1]. PTX3 is structurally related but distinct from the classic short pentraxins, C-reactive protein and serum amyloid protein, differing in gene organization and localization, ligand recognition, producing cells, and inducing signals. PTX3 is expressed in macrophages, dendritic cells, neutrophils, and vascular endothelial cells (ECs), but not in the liver, in response to primary proinflammatory signals such as bacterial products, interleukin-1, and tumor necrosis factor-alpha, but not interleukin-6 [2-5]. PTX3 level is known to be increased in the plasma of patients with acute coronary syndrome (ACS) $[6,7]$, but the mechanism causing the increase remains unknown. Recently we have developed a highly sensitive ELISA system for the measurement of human PTX3 in plasma using high-specificity monoclonal antibodies [8]. The assay has a coefficient of variation of $4.1 \%$, and the sensitivity is 7 times higher than that of commercially available kits. Using our assay, we have detected high plasma PTX3 levels in patients with unstable angina pectoris.

The present study compared plasma PTX3 levels at the site of ruptured plaque and the aorta in patients with ACS and investigated thrombi from patients with ACS to elucidate the mechanism of increased plasma PTX3 level in patients with ACS. 


\section{Methods}

2.1. Patient Population. A total of 720 patients, 551 men and 169 women aged 21 to 94 years (mean age $66.8 \pm$ 10.2 years), were admitted to Juntendo University Hospital, Juntendo University Shizuoka Hospital, or Juntendo University Nerima Hospital for assessment of ischemic heart disease, including ACS, arteriosclerosis obliterans (ASOs), or vasospastic angina pectoris (VAP) by coronary angiography (CAG) between January 2006 and June 2007. The diagnosis of ACS was based on the presence of more than one clearly identifiable culprit lesion by CAG in patients with electrocardiography findings of diagnostic ST-segment changes or T-wave inversion with chest pain.

ASO was defined by stable intermittent claudication stage II according to the Fontaine classification and by angiography findings of severe stenosis of the iliac or suprafemoral artery. VAP was defined as angina precipitated by resting or sleeping associated with ST segment elevation on electrocardiography, but no severe focal stenosis in the coronary arteries by CAG. The normal value of plasma PTX3 was established in 1739 subjects who underwent annual health exams in 2006 in the area of Kamigoto, a suburb of Nagasaki city in Southern Japan, as reported previously [9]. From these data, 709 age-matched subjects were selected. Study exclusion criteria were described previously [8]. Briefly, we excluded patients with chronic inflammation status or any history of inflammatory disease in the one month prior to admission, due to the fact that levels of C-reactive protein often remain elevated. We also excluded patients with renal insufficiency or malignant tumors. Finally the study investigated 698 subjects, 539 men and 159 women aged 21 to 91 years (mean age $66.7 \pm 10.1$ years), including 129 patients with ACS, 26 patients with VAP, and 37 patients with ASO. All patients were of Japanese nationality and gave informed consent. This study was approved by the ethical committee of Juntendo University and conducted in accordance with the Helsinki Declaration of 1971, as revised in 1983.

2.2. Aspiration Catheter. The Rebirth Aspiration catheter (Goodman Co., Ltd., Aichi, Japan) is a thrombectomy system consisting of a $4.5 \mathrm{~F}$ polyethylene catheter to be advanced over a guidewire through a $6 \mathrm{~F}$ guiding catheter. The proximal end of the catheter has an extension tube connected to a $25 \mathrm{mlL}$ syringe. The catheter was advanced to the culprit lesion, and continuous suction was performed. The present study collected samples as follows: before percutaneous coronary intervention, blood samples were collected from the aorta using a guiding catheter (Sample 1); after inserting the guiding catheter into the coronary artery, the aspiration catheter was advanced to the culprit lesion, and the thrombi, along with a blood sample, were collected (Sample 2).

2.3. Laboratory Measurements. Plasma total cholesterol, high- and low-density lipoprotein cholesterol, triglyceride, hemoglobin A1C, and brain natriuretic peptides (BNP) were measured. Plasma PTX3 levels were measured using a human
PTX3 ELISA kit (Perseus Proteomics Inc.) as previously reported [8]. In each case, $4 \mathrm{~mL}$ of blood was drawn into an EDTA vacuum container for PTX3 measurement and frozen at $-20^{\circ} \mathrm{C}$ until time of assay. Blood samples were obtained immediately before CAG following an overnight fast.

\subsection{Aspirated Thrombi Specimens from the Coronary Artery.} Aspirated thrombi from patients with acute myocardial infarction (AMI) $(n=32)$ were examined by histological staining. Thrombi were fixed for 1 day at room temperature in $15 \%$ formalin, sequentially dehydrated with an alcohol series, and embedded in paraffin. The sections were stained with hematoxylin and eosin.

2.5. Immunohistochemistry and Antibodies. Histochemical staining for nonspecific esterase and immunohistochemical staining employing primary monoclonal antibodies against CD15 or macrophage scavenger receptor-class A (CD204) antibody were used to define neutrophils or macrophages, respectively. Anti-human PTX3 monoclonal antibody (clone 1228) was provided by Perseus Proteomic Inc. The protocol was described previously [4].

2.6. Quantitative Methods. Numbers of anti-CD15-positive neutrophils and anti-PTX3 antibody-positive cells were counted in the entire tissue sections and expressed as the number of cells per square millimeter of tissue. The morphometric analysis was performed by a single investigator who was unaware of the patients' characteristics. Values are shown as the mean $\pm \mathrm{SD}$.

2.7. Statistical Analysis. Statistical analysis was performed using the SPSS software package (SPSS 18.0; SPSS Inc., Chicago, IL, USA). Parametric tests such as analysis of variance (ANOVA) were used after log-transformation of the original data, because PTX3 values did not show a normal distribution but approximated a log-normal distribution. The paired $t$-test was used for comparison of the two groups. One-way ANOVA with the Bonferroni test was used to identify differences among the 4 groups. Values are expressed as the mean \pm SD. PTX3 and BNP values are expressed as the arithmetic mean values \pm SD. $P$ values of $<0.05$ were considered to be statistically significant. Ratios and proportions were compared between different classes using the chi-square test.

\section{Results}

3.1. Plasma PTX3 Levels Not Increased in Patients with VAP or ASO. To investigate whether elevated plasma PTX3 levels are specific to patients with ACS, plasma PTX3 levels were measured in patients with other atherosclerotic diseases, including ASO or VAP. Table 1 lists the characteristics of the disease groups. Plasma PTX3 levels showed no significant differences between the ASO group $(2.33 \pm 1.61 \mathrm{ng} / \mathrm{mL})$, the VAP group $(2.16 \pm 0.86 \mathrm{ng} / \mathrm{mL})$, and the normal agematched population. Therefore, atherosclerosis except ACS did not seem to increase plasma PTX3 levels. 


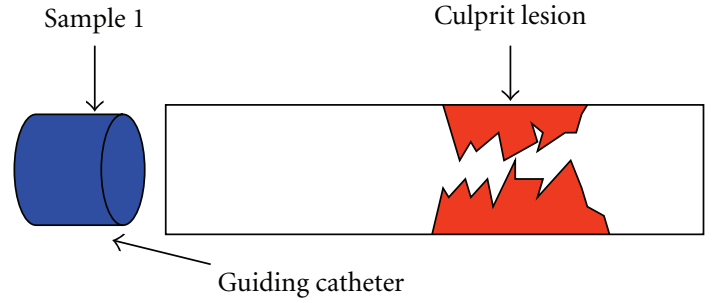

(a)

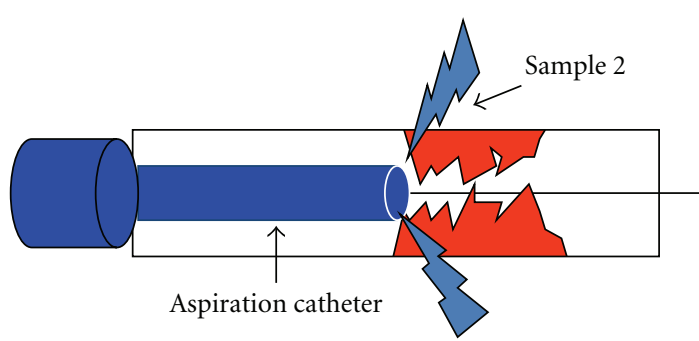

(b)

FIGURE 1: Schema showing how to collect Sample 1 and Sample 2. Sample 1 was taken from the guiding catheter before insertion into the coronary artery (a), and Sample 2 was taken from the aspiration catheter (b).

TABLE 1: Characteristics of patients in ACS, VAP, or ASO group.

\begin{tabular}{|c|c|c|c|c|}
\hline & Normal & ACS & VAP & ASO \\
\hline Number & 709 & 129 & 26 & 37 \\
\hline Age, yr & $67.7 \pm 4.5$ & $67.8 \pm 12.7$ & $62.2 \pm 9.06$ & $8.1 \pm 7.7$ \\
\hline Male sex, no. (\%) & 46.8 & 71.0 & 73.1 & 85.7 \\
\hline Smoker, no. (\%) & Not available & 81.1 & 63.6 & 89.7 \\
\hline $\mathrm{BMI}\left(\geqq 24.2 \mathrm{~kg} / \mathrm{m}^{2}\right)$, no $\%$ & 23.9 & 31.8 & 27.2 & 33.3 \\
\hline $\mathrm{TCHO}, \mathrm{mg} / \mathrm{dL}$ & $208.0 \pm 32.8$ & $189.5 \pm 39.9$ & $189.3 \pm 37.4$ & $201.3 \pm 50.8$ \\
\hline LDL-c, mg/dL & $122.1 \pm 28.6$ & $124.8 \pm 36.2$ & $103.6 \pm 16.6$ & $127.8 \pm 30.9$ \\
\hline HDL-c, mg/dL & $65.8 \pm 16.3$ & $49.3 \pm 12.2$ & $61.9 \pm 14.6$ & $45.0 \pm 11.1$ \\
\hline $\mathrm{TG}, \mathrm{mg} / \mathrm{dL}$ & $108.1 \pm 45.3$ & $137.3 \pm 63.8$ & $84.2 \pm 34.4^{*}$ & $141.1 \pm 59.1$ \\
\hline HgbA1C, \% & $5.5 \pm 0.6$ & $6.0 \pm 1.7$ & $5.1 \pm 0.4$ & $6.1 \pm 1.1$ \\
\hline \multicolumn{5}{|l|}{ Plasma PTX3 (ng/mL) } \\
\hline Arithmetic means & $2.11 \pm 1.60$ & $5.80 \pm 5.33^{*}$ & $2.16 \pm 0.86$ & $2.33 \pm 1.61$ \\
\hline
\end{tabular}

ACS: acute coronary syndrome; VAP: vasospastic angina pectoris; ASO: arteriosclerosis obliterans;BMI: body mass index; TCHO: total cholesterol; LDL-c: lowdensity lipoprotein cholesterol; HDL: high-density lipoprotein cholesterol; TG: triglyceride; HgbA1C: hemoglobin A1C; PTX3: pentraxin $3 .{ }^{*} P<0.00001$.

3.2. Plasma PTX3 Levels at the Site of Culprit Lesions. The major difference between patients with ACS and patients with ASO or VAP is the presence of thrombi at the sites of plaque rupture. Therefore, we investigated whether these sites are responsible for the increased levels of PTX3 observed in patients with ACS. We collected blood samples from the aorta and culprit lesions in coronary arteries containing ruptured plaques in patients with ACS using a guiding and an aspiration catheter (Figure 1). Thrombi were aspirated during percutaneous coronary intervention from 118 of the 129 patients with ACS. As a reference standard in this investigation, we measured plasma BNP levels in both Samples 1 and 2, as BNP is known to be released from ventricular cardiomyocytes, but not from plaque $[10,11]$. The level of plasma BNP did not correlate with the aspiration site as plasma BNP levels were higher in aorta samples (Sample 1) compared to the ruptured site (Sample 2) in 51 subjects, whereas 63 subjects showed higher levels in Sample 1 compared to Sample 2, and 4 subjects showed similar values in both samples. Plasma BNP levels showed no difference between Sample 1 and $2(92.24 \pm 256.53 \mathrm{pg} / \mathrm{mL}$ versus $120.9 \pm 287.14 \mathrm{pg} / \mathrm{mL}, P=0.59$ ) (Figure $2(\mathrm{a})$ ). In contrast, plasma PTX3 levels were significantly higher in blood samples from the site of ruptured plaque (Sample 2) compared to blood samples taken from the aorta (Sample1) $(5.61 \pm 1.91 \mathrm{ng} / \mathrm{mL}$ versus $4.72 \pm 5.61 \mathrm{ng} / \mathrm{mL}, P<0.05)$ in
97 patients $(82.2 \%)$, suggesting that cells in the culprit lesion released PTX3 (Figure 2(b)).

3.3. Localization of PTX3 in Neutrophils Infiltrating Thrombi. To further investigate the possibility that PTX3 is produced and released from superimposed thrombi, PTX3 expression patterns were immunohistochemically analyzed in thrombi obtained using an aspiration catheter from 32 patients. To identify the cells responsible for PTX3 expression, serial staining was performed for PTX3 and specific cell types, including macrophages or neutrophils. Figure 3 showed representative data obtained from a 67year-old man with AMI. Although a number of cells had infiltrated the thrombus (Figure 3(a)), the overwhelming majority were neutrophils and mononuclear cells, with only a few macrophages (Figure 3(d)). PTX3 was expressed in polynuclear cells (Figures 3(b) and 3(e)) stained by antiCD15 antibody, a known marker for neutrophils (Figures 3(c) and 3(f)). PTX3-positive neutrophils accounted for $70.4 \%$ of cells in the examined thrombi (Figure 4 ), with the remaining cells consisting predominantly of mononuclear cells. The major difference between patients with ACS and patients with ASO or VAP is the presence of thrombi at the sites of plaque rupture. Activated or injured ECs are present in atherosclerotic lesions in patients with ASO or VAP and 


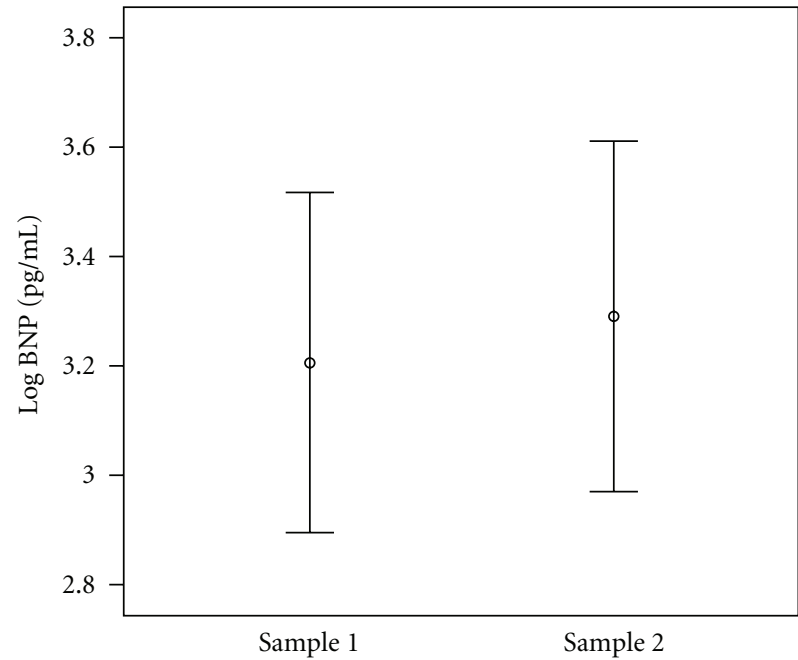

(a)

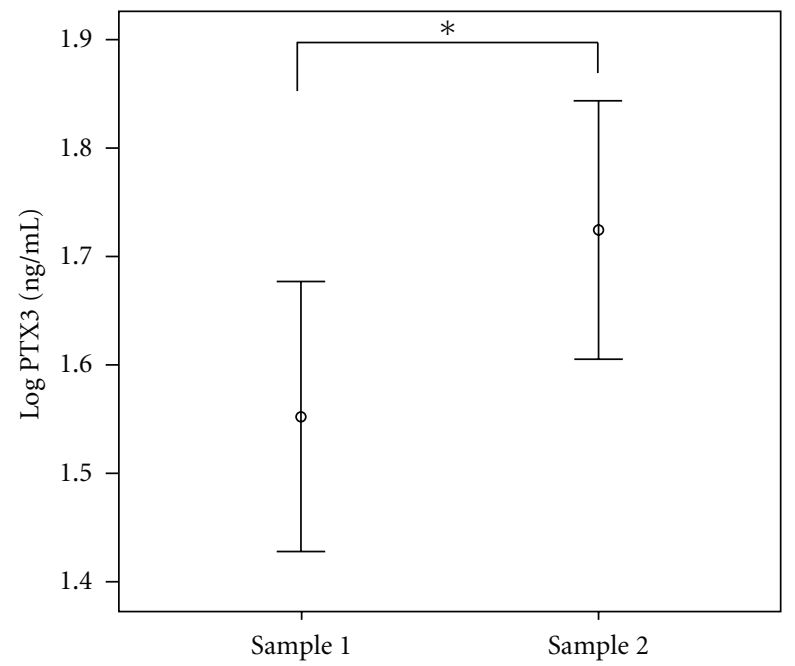

(b)

FIGURE 2: Comparison of plasma PTX3 or BNP levels in blood aspirated from the aorta (Sample 1) and the plaque site (Sample 2). Plasma BNP levels showed no difference between Samples 1 and $2(92.24 \pm 256.53 \mathrm{pg} / \mathrm{mL}$ versus $120.9 \pm 287.14 \mathrm{pg} / \mathrm{mL}, P=0.59$ (a)), but plasma PTX3 was significantly lower in Sample 1 than in Sample $2(4.72 \pm 5.61 \mathrm{ng} / \mathrm{mL}$ versus $5.61 \pm 1.91 \mathrm{ng} / \mathrm{mL}, P<0.05$ (b)).

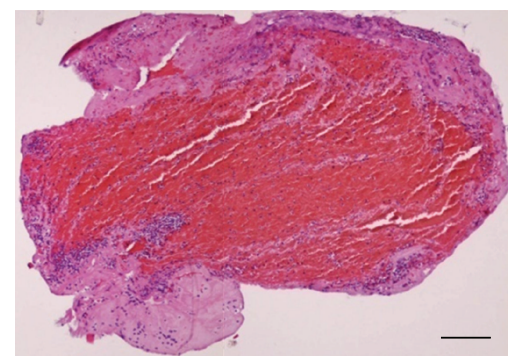

(a)

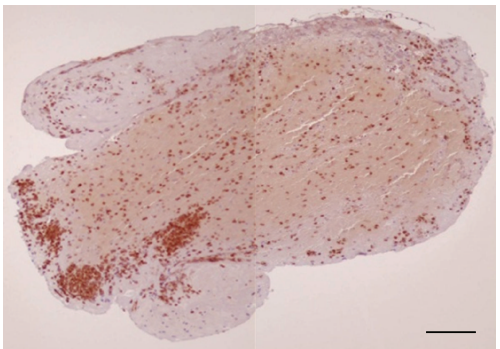

(c)

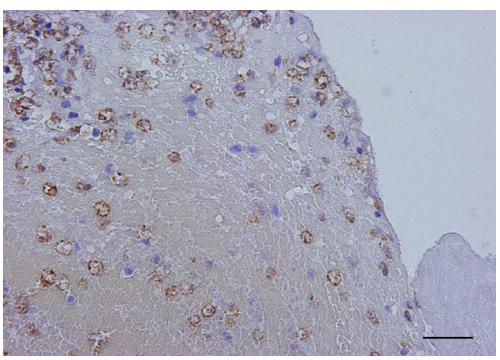

(e)

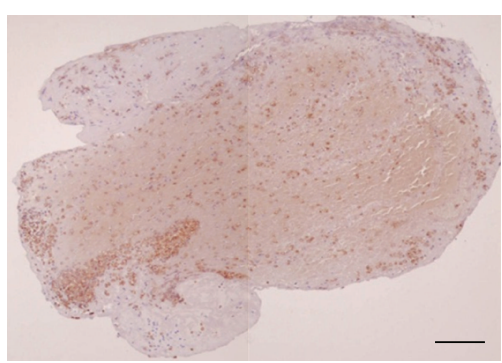

(b)

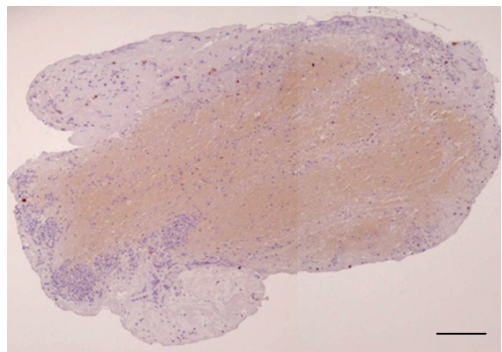

(d)

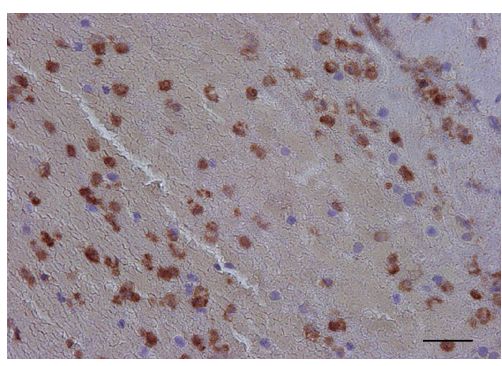

(f)

FIGURE 3: Expression patterns of inflammatory cells and PTX3 in the thrombus obtained from a 67-year-old man with AMI. Inflammatory cells infiltrated around the red thrombus ( (a) hematoxylin and eosin, $\times 200)$. The expression patterns of CD15-positive cells were similar for neutrophils ((b) $\times 200$ and (e) $\times 400)$ and PTX3-positive cells $((\mathrm{c}) \times 200$ and $(\mathrm{f}) \times 400)$, but negative staining for CD204 suggested macrophages $((\mathrm{d}) \times 200)$. Bar indicates $200 \mu \mathrm{m}$. Bar indicates $50 \mu \mathrm{m}$. 


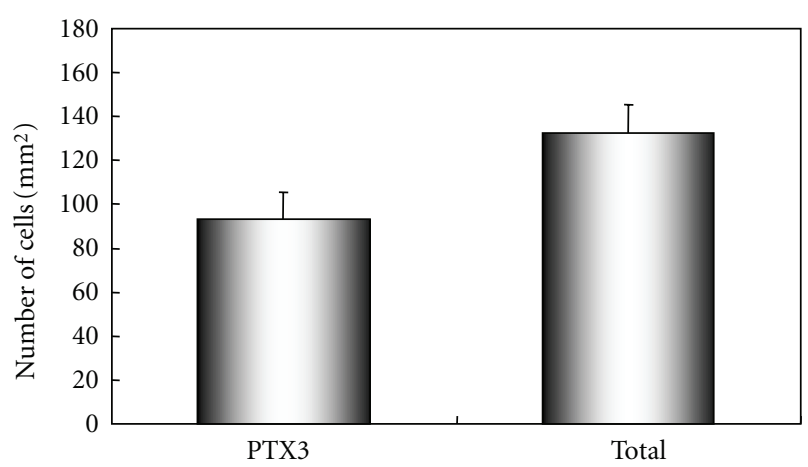

Figure 4: Distribution patterns of cells in thrombi from patients with ACS. $Y$-axis indicated the number of cells per square millimeter. Graph showing the number of PTX3-positive total cells $/ \mathrm{mm}^{2}$ in the thrombus obtained from the culprit lesion in patients with ACS $(n=32)$. PTX3-positive neutrophils accounted for $70.4 \%$ of cells in the examined thrombi.

activated macrophages in patients with ASO, but the plasma PTX3 level was almost the same as in normal subjects. Collectively, these results strongly suggest that neutrophils in thrombi formed over the ruptured plaque site are one of the most important sources of PTX3.

\section{Discussion}

The present study demonstrated that PTX3 originates from neutrophils at the site of plaque rupture in the coronary artery of patients with ACS. Plasma PTX3 levels are increased in patients with AMI [6], and we previously suggested that PTX3 may represent a good biomarker for the diagnosis of patients with unstable angina pectoris [8]. This study demonstrated that plasma PTX3 levels were not increased in patients with ASO or VAP. Our findings indicate that plaque volume or endothelial dysfunction may not be important to increase plasma PTX3 level. Plaque disruption with superimposed occlusive or nonocclusive thrombosis is the main cause of ACS, in contrast to ASO or VAP $[12,13]$. In this study, the investigation of blood samples collected by aspiration catheter revealed that $82.2 \%$ of ACS patients exhibited significantly higher levels of PTX3 in blood samples taken from the plaque site in the coronary artery compared to samples taken from the aorta.

Immunohistochemical examination of aspirated thrombi demonstrated the infiltration of neutrophils expressing PTX3 [4]. This study found that almost $70 \%$ of the neutrophils expressed PTX3 in the thrombus (Figure 3). The remaining cells were monocytes which did not express PTX3. PTX3 is stored in specific granules, neutrophil extracellular traps, which are rapidly secreted after stimulation [14, 15]. Therefore, the PTX3 stored in the granules will rapidly enter the blood. Direct release of PTX3 from ECs or macrophages has not been demonstrated. If ECs or macrophages are the main sources to release PTX3, plasma PTX3 should be increased in patients with VAP or ASO. All thrombi from patients with AMI contain large numbers of neutrophils [16]. The leukocytes identified within the coronary artery lumen are primarily neutrophils, suggesting the presence of inflammation within the coronary artery in patients with ACS. ACS is usually asymptomatic for a long period of time, with repeat small or minor plaque ruptures, finally culminating in thrombosis. Our findings suggest that PTX3 levels might provide an excellent marker of these events.

The present study demonstrated that plasma PTX3 levels were increased in patients with ACS, but not in patients with ASO or VAP, probably caused by the infiltration of PTX3expressing neutrophils. Our findings suggest that PTX3, or diagnostically significant elevation in a patient's PTX3 level, predominantly originates from neutrophils infiltrating the thrombus at sites of plaque rupture.

\section{Acknowledgments}

The authors would like to thank Dr. Patrick C. Reid for his critical discussion and reading the paper carefully. They also would like to thank Noriko Hirose and Yuri Naito for technical support. K. Inoue and S. Suwa contributed equally. This study was supported by the Program of Fundamental Studies in Health Sciences of the NIBIO, NEDO, and by the Fund for Science and Technology from the Ministry of Education, Culture, Sports, Science and Technology in Japan.

\section{References}

[1] F. Breviario, E. M. D’Aniello, J. Golay et al., "Interleukin1-inducible genes in endothelial cells. Cloning of a new gene related to C-reactive protein and serum amyloid $\mathrm{P}$ component," Journal of Biological Chemistry, vol. 267, no. 31, pp. 22190-22197, 1992.

[2] V. V. Alles, B. Bottazzi, G. Peri, J. Golay, M. Introna, and A. Mantovani, "Inducible expression of PTX3, a new member of the pentraxin family, in human mononuclear phagocytes," Blood, vol. 84, no. 10, pp. 3483-3493, 1994.

[3] B. Bottazzi, V. Vouret-Craviari, A. Bastone et al., "Multimer formation and ligand recognition by the long pentraxin PTX3. Similarities and differences with the short pentraxins Creactive protein and serum amyloid $\mathrm{P}$ component," Journal of Biological Chemistry, vol. 272, no. 52, pp. 32817-32823, 1997.

[4] A. S. Savchenko, M. Imamura, R. Ohashi et al., "Expression of pentraxin 3 (PTX3) in human atherosclerotic lesions," Journal of Pathology, vol. 215, no. 1, pp. 48-55, 2008.

[5] M. S. Rolph, S. Zimmer, B. Bottazzi, C. Garlanda, A. Mantovani, and G. K. Hansson, "Production of the long pentraxin PTX3 in advanced atherosclerotic plaques," Arteriosclerosis, Thrombosis, and Vascular Biology, vol. 22, no. 5, pp. e10-14, 2002.

[6] R. Latini, A. P. Maggioni, G. Peri et al., "Lipid Assessment Trial Network (LATIN) Investigators. Prognostic significance of the long pentraxin PTX3 in acute myocardial infarction," Circulation, vol. 110, no. 16, pp. 2349-2354, 2004.

[7] G. Peri, M. Introna, D. Corradi et al., "PTX3, a prototypical long pentraxin, is an early indicator of acute myocardial infarction in humans," Circulation, vol. 102, no. 6, pp. 636$641,2000$.

[8] K. Inoue, A. Sugiyama, P. C. Reid et al., "Establishment of a high sensitivity plasma assay for human pentraxin3 as a marker for unstable angina pectoris," Arteriosclerosis, 
Thrombosis, and Vascular Biology, vol. 27, no. 1, pp. 161-167, 2007.

[9] K. Yamasaki, M. Kurimura, T. Kasai, M. Sagara, T. Kodama, and K. Inoue, "Determination of physiological plasma pentraxin 3 (PTX3) levels in healthy populations," Clinical Chemistry and Laboratory Medicine, vol. 47, no. 4, pp. 471477, 2009.

[10] O. Nakagawa, Y. Ogawa, H. Itoh et al., "Rapid transcriptional activation and early mRNA turnover of brain natriuretic peptide in cardiocyte hypertrophy. Evidence for brain natriuretic peptide as an "emergency" cardiac hormone against ventricular overload," Journal of Clinical Investigation, vol. 96, no. 3, pp. 1280-1287, 1995.

[11] K. Maeda, T. Tsutamoto, A. Wada, T. Hisanaga, and M. Kinoshita, "Plasma brain natriuretic peptide as a biochemical marker of high left ventricular end-diastolic pressure in patients with symptomatic left ventricular dysfunction," American Heart Journal, vol. 135, no. 5 I, pp. 825-832, 1998.

[12] V. Fuster, L. Badimon, J. J. Badimon, and J. H. Chesebro, “The pathogenesis of coronary artery disease and the acute coronary syndromes," The New England Journal of Medicine, vol. 326, no. 5, pp. 242-250, 1992.

[13] V. Fuster, P. R. Moreno, Z. A. Fayad, R. Corti, and J. J. Badimon, "Atherothrombosis and high-risk plaque: part I: evolving concepts," Journal of the American College of Cardiology, vol. 46, no. 6, pp. 937-954, 2005.

[14] S. Jaillon, G. Peri, Y. Delneste et al., "The humoral pattern recognition receptor PTX3 is stored in neutrophil granules and localizes in extracellular traps," Journal of Experimental Medicine, vol. 204, no. 4, pp. 793-804, 2007.

[15] S. Jaillon, P. Jeannin, Y. Hamon et al., "Endogenous PTX3 translocates at the membrane of late apoptotic human neutrophils and is involved in their engulfment by macrophages," Cell Death and Differentiation, vol. 16, no. 3, pp. 465-474, 2009.

[16] Y. Nagata, K. Usuda, A. Uchiyama et al., "Characteristics of the pathological images of coronary artery thrombi according to the infarct-related coronary artery in acute myocardial infarction," Circulation Journal, vol. 68, no. 4, pp. 308-314, 2004. 


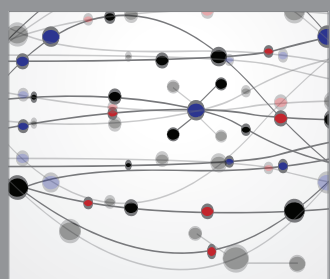

The Scientific World Journal
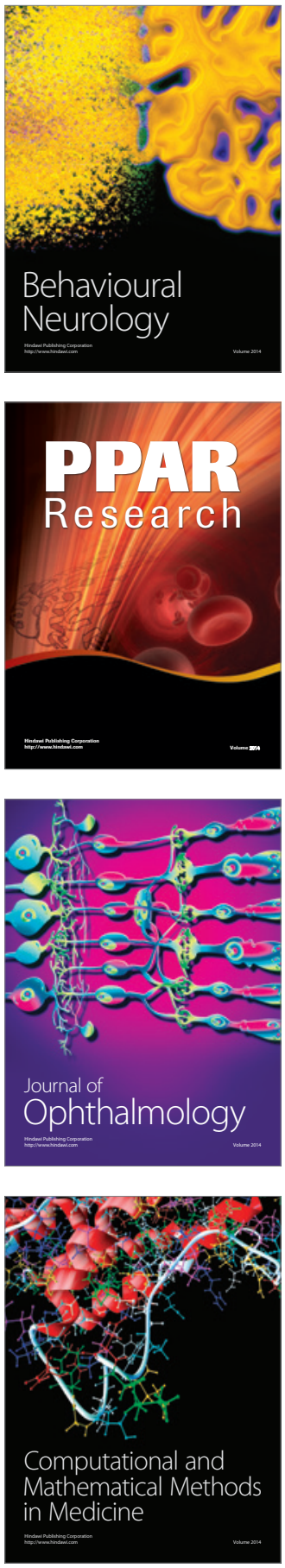

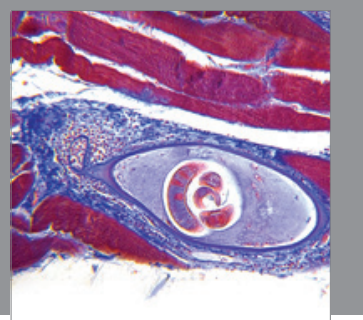

Gastroenterology

Research and Practice
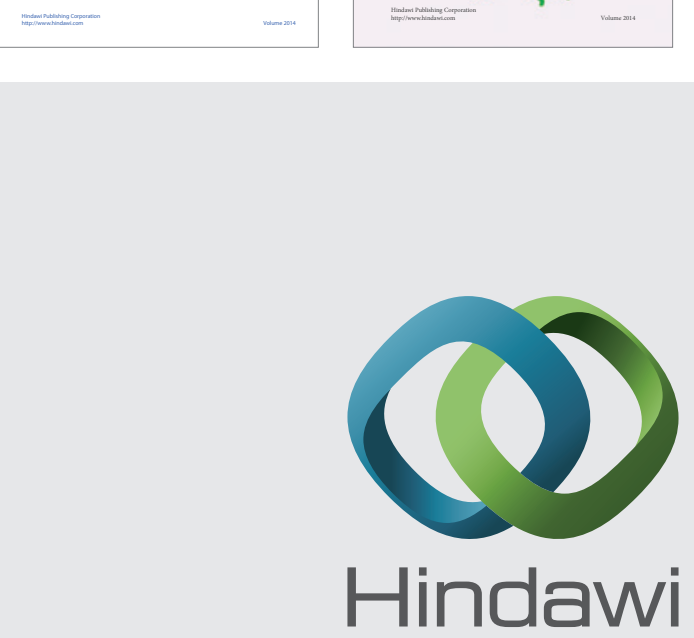

Submit your manuscripts at

http://www.hindawi.com
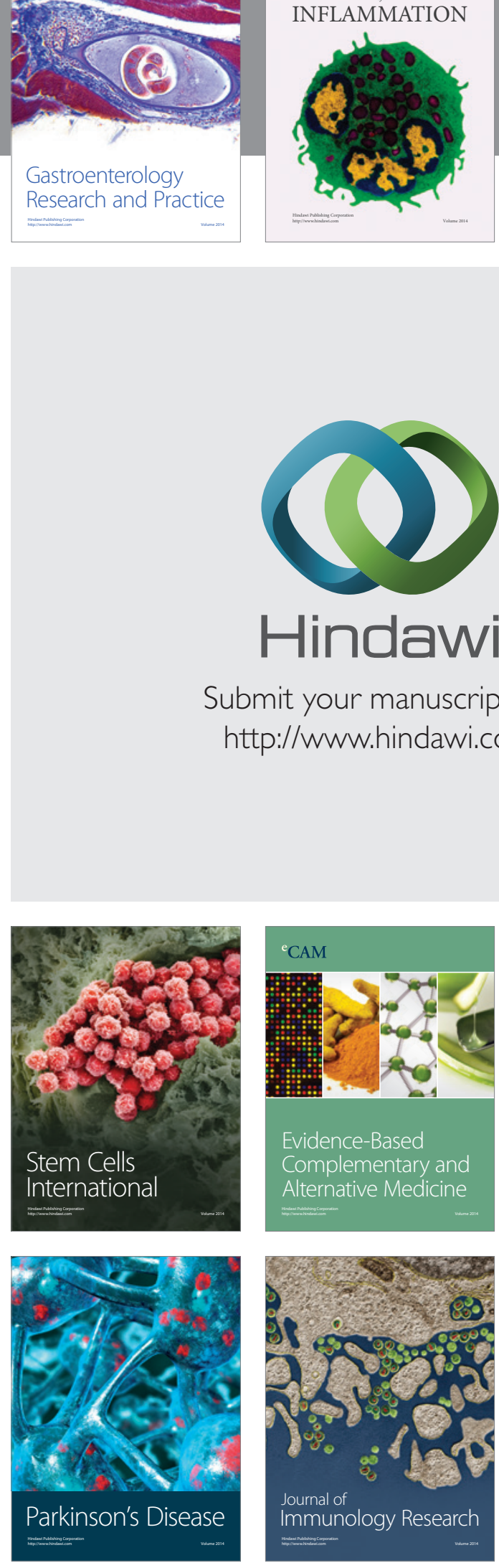

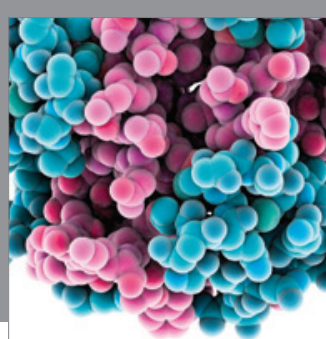

Diabetes Research
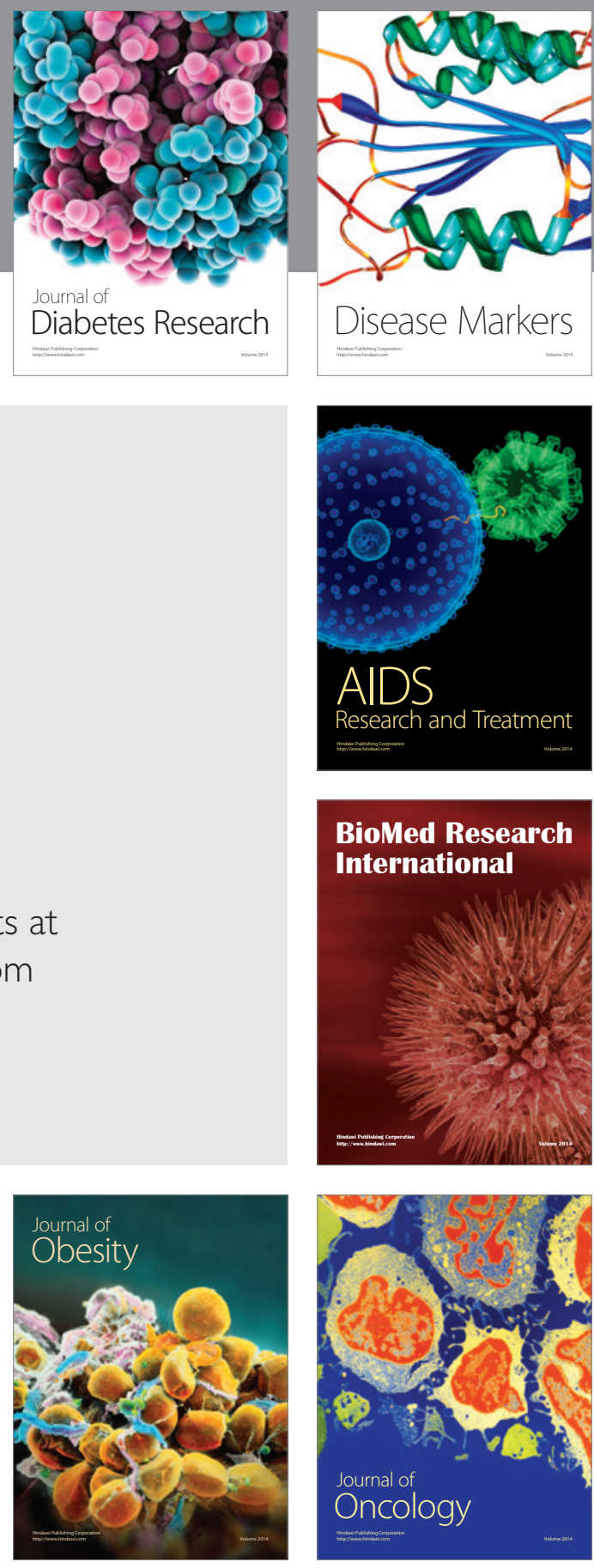

Disease Markers

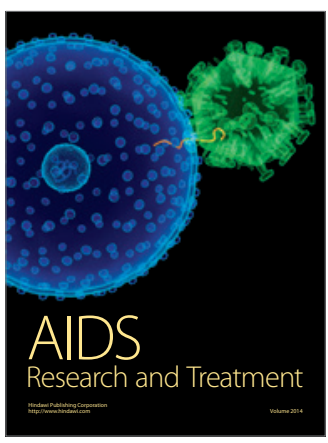

BioMed Research

International
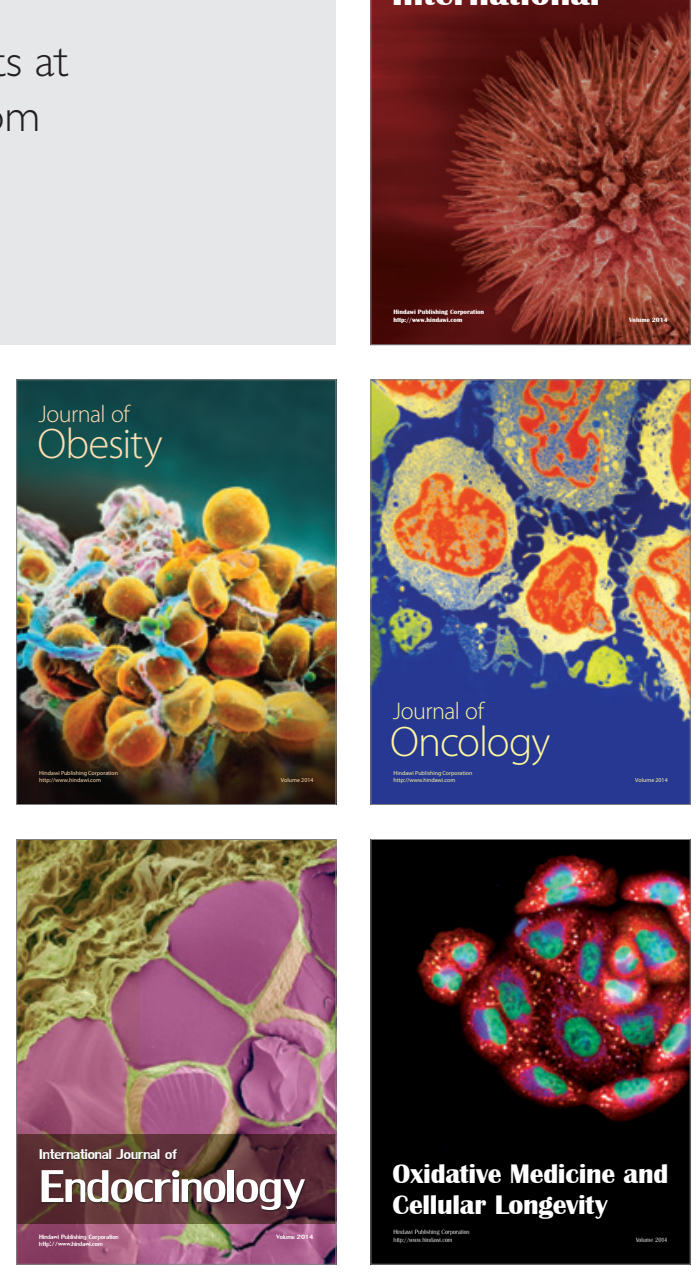